\title{
Semi-Markov model for the oxidation degradation mechanism in
} gas turbine nozzles

\author{
M.Compare' , F. Martini' ${ }^{2}$, S. Mattafirri ${ }^{3}$, F. Carlevaro ${ }^{3}$, E. Zio Zi,2, $^{1,4}$ \\ ${ }^{1}$ Aramis s.r.l., Milano, Italy* \\ ${ }^{2}$ Dipartimento di Energia, Politecnico di Milano, Italy \\ ${ }^{3}$ General Electric-Nuovo Pignone, Italy \\ ${ }^{4}$ Chair on Systems Science and Energetic Challenge, European Foundation for New Energy-Electricité de \\ France, Ecole Centrale Paris and Supelec, Paris, France \\ *corresponding author: michele.compare@aramis3d.com
}

\begin{abstract}
This paper presents the statistical characterization of the oxidation degradation mechanism affecting the nozzles of turbines operated in Oil\&Gas utilities. The degradation mechanism is modelled as a four-state, continuous-time semi-Markov process with Weibull distributed transition times. Maximum Likelihood Estimation is used to infer the parameters of the model from an available set of field data, whereas a numerical approach to estimate the Fisher information matrix is used to characterize the uncertainty in the estimates. The estimates obtained are, then, utilized to compute the probabilities of occupying the four degradation states over time and the corresponding uncertainties. A case study is shown, dealing with real field data.
\end{abstract}

\section{Introduction}

Multi-state degradation modelling has recently received considerable attention in the domain of reliability and maintenance engineering (e.g., [1]-[10]). There are several reasons to justify such a growing interest. First, multi-state models offer more granularity for tracing the evolutions of the degradation phenomena more precisely than binary models, which "lump" the degradation process description into just two (extreme) states, 'good' and 'failed': indeed, such binary-state assumption is not adequate in most real-life situations. For example, system consisting of different units that have a cumulative effect on the entire system performance has to be modelled as a Multi-State system [11]. Indeed, the performance rate of the overall system depends on the availability of its units, as the different numbers of the available units can provide different levels of task performance.

Also, multi-state degradation models offer more coherence with the descriptive maintenance data acquired on the operating systems. Maintenance teams in different industrial fields often assign a 
qualitative tag to describe the current health condition in which equipment is found at inspection. Thus, multi-state modelling is adopted by maintenance engineers to describe, for example, the evolution of degradation processes affecting components of the electrical industry [1]-[2], the membranes of pumps operating in nuclear power plants [4], [5]. In this study, we propose a case study from the Oil\&Gas industry.

Furthermore, the evolution of some degradation processes can often be sub-divided into successive phases, characterized by physically different degradation mechanisms. This suggests adopting a multi-state model to describe the degradation progression. In this respect, the creep mechanism affecting the blades of turbines, the degradation in piping systems of nuclear power plants, etc., are typical examples of four-state degradation models. Every state corresponds to a different physical behaviour. For example, primary, secondary and tertiary creep, and failure are the different phenomena experienced by turbine blades [12], whereas no damage, micro-crack, flaw and rupture are the states that give rise to physically different mechanisms on pipes [13], [14].

Multi-state modelling is also useful in support of the setting of advanced maintenance paradigms, such as Condition-Based Maintenance (CBM) and Predictive Maintenance (PrM, [15], [16]). Indeed, the description by multiple states offers different alternatives to define thresholds at which the equipment needs to undergo different types of maintenance care: this setting is at the basis of any CBM approach. Moreover, the equipment Residual Useful Life (RUL) can be supported by multistate modelling and this gives the opportunity for PrM with potential for improvement in the organization of the maintenance and workshop activities, logistics, spare part management, etc. [6], [7].

The characterization of the stochastic transitions among the states is fundamental in multi-state modelling. In this work, we assume that the transitions can occur from one state to the next degraded state, only, which is typical of degradation mechanisms driven by cumulative damage, with no shocks. In fact, cumulative degradation processes successively visit the degradation states and jumps of more than one degradation step.

Moreover, the transition times are assumed to obey Weibull distributions. From the engineering point of view, this choice is justified by the flexibility of the Weibull distribution, which allows encoding hazard functions both increasing and decreasing over time, with different speeds. From the mathematical point of view, this translates in describing the transition processes among the states, which do not follow the memory-less property of Markov processes (i.e., exponential-distributed transition times): the probability that an equipment, which has spent $X$ time units in the $i$-th state, will 
spend an additional time $Y$, depends only on $Y$ (not on $X$ ), and is identical to the probability of remaining $Y$ time units in the same $i$-th state of an equipment just arrived in that state [17]. Certainly, this property does not apply to mechanical systems, which suffer from aging and/or infant mortality. On the contrary, the Weibull distribution accounts for the dependence of the state-departing transition time on the time spent in the state, thus offering a way to describe the degradation behaviour which is more realistic. Then, the multi-state model we propose for describing the degradation mechanism can be framed as a semi-Markov process.

The model used to describe the transitions among the multi states, relies on parameters (e.g., scale and shape factors, failure rates, etc.) whose values must be inferred from the available data. The Maximum Likelihood Estimation (MLE) is a well-known statistical technique, commonly used to estimate parameters of the probability distributions [18]. However, in multi-state models when the number of states becomes large (e.g., $\geq 4$ ) and their transition times are not exponentially distributed, the application of the MLE technique is not straightforward, as it requires solving complex integrals which encode the probabilities associated with all the possible states (e.g., outcomes of the turbine visual inspections after a given working time, in our case study).

In this work, we develop a numerical approach to solve the MLE problem for multi-state degradation models, which combines the numerical computation of the involved integrals with an optimization algorithm to find the maximum of the log-likelihood function. Moreover, a procedure is proposed for characterizing the uncertainty in the MLE parameters, which is based on a bootstrap technique [19] to estimate the Fisher Information Matrix (FIM, [18]). Finally, the ML estimate and the estimated FIM are used to propagate the uncertainty onto the probabilities of occupying the possible degradation states over time.

The results of the methodology are fundamental for the end users of the degradation model, who are decision makers interested in taking robust business decisions by calculating the economic risk associated with a given maintenance service contract, based on the system life cycle cost. In fact, the degradation model can be embedded into a PrM or CBM maintenance model to estimate the performance indicators of interest (e.g., unavailability, profitability, quality in production, total costs, etc.), with the corresponding uncertainty. These outcomes are at the basis of the optimization algorithm, which identifies the set of optimal maintenance settings in the presence of uncertainty ([20], [21]). On this basis, the decision maker selects the preferred maintenance solution. 
The proposed methodology is applied to a practical case study concerning the oxidation mechanism affecting the inner part of the nozzles of gas turbines operated in energy production plants by General Electric (GE).

The contribution of the work is threefold: first, it provides a comprehensive development pathway to build a semi-Markov degradation model, from the estimation of its parameters up to the estimation of the probabilities of occupying the states in time, together with corresponding uncertainties; then, it paves the way for the development and use of computational methods to optimize CBM strategies in presence of uncertainty ([20]-[21]); finally, it illustrates an application of the multi-state modeling approach to a real industrial application concerning the nozzle system of Gas Turbines (GTs), which represents a novelty in that specific domain.

The remainder of the paper is organized as follows. Section 2 describes the considered degradation model and its assumptions, Section 3 proposes a numerical algorithm to estimate the parameters of the model, whereas in Section 4 bootstrap is applied to estimate the uncertainty in the parameter estimates. The application of the methodology to a GE gas turbine is shown and discussed in Section 5. Concluding remarks are given in Section 6.

\section{Model Assumptions}

In this work, we consider a four-state degradation process (Figure 1), where:

- State 'Good' refers to a new degraded component, and it is indicated by $S_{1}$

- State 'Light' refers to a mildly degraded component, and it is indicated by $S_{2}$

- State 'Medium' refers to a fairly degraded component, and it is indicated by $S_{3}$

- State 'Heavy' refers to a heavily degraded component, and it is indicated by $S_{4}$.

As mentioned before, it is assumed that direct transitions from state $S_{1}$ directly to state $S_{3}$ or $S_{4}$ are not possible, nor are the transitions from state $S_{2}$ to state $S_{4}$ (Figure 1). As explained in the Introduction, this is typical of the cumulative degradation processes, which successively visit all the states.

The stochastic transition times from state $k$ to $k+1$ are indicated by $T_{k}, k=1,2,3$. These are assumed to obey Weibull distributions with scale and shape parameters $\alpha_{k}$ and $\beta_{k}$, respectively.

It is also assumed that a dataset of inspection outcomes is available, which is used to estimate the model parameters. In this respect, we model the inspection time as a random variable statistically independent from the transition times of the degradation model. This assumption, which is usually referred to as non-informative censoring (e.g., [23]), implies that the choice of the inspection time for 
each component in the dataset is not influenced by any information collected during the component life (ongoing detections, measurements, etc.), which could have revealed, directly or indirectly, the evolution of its degradation state. The justification of such an assumption again relates to the specific, real case study considered in this paper (for further details, see [24]).

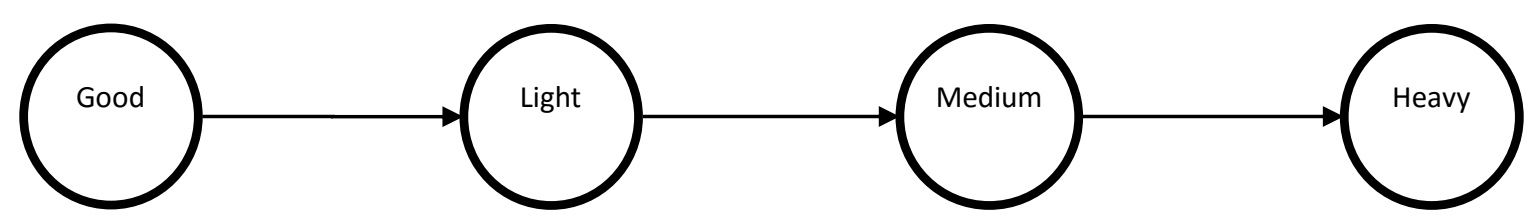

Figure 1: Scheme of the degradation mechanism

We assume that $N$ inspections are conducted during the study period and $n$ components are inspected each time. The $i$-th inspection outcome reports the degradation states of these $n$ components, which have behaved independently of each other. Thus, the available dataset of inspection outcomes is made up of $N$ records, each one containing (Table 1):

1. The time at which the system has been inspected.

2. The number of components found in state $S_{s}$, (i.e., $\left.n_{s}\right) s=1, \ldots, 4$, where $n=\sum_{s=1}^{4} n_{s}$.

Finally, we assume that upon inspection, the $n$-components system is removed from operation, to be submitted to the proper repair actions, which depend on the degradation states of the individual components. These actions are assumed to always bring the system back to an As Good As New (AGAN) state. This assumption is made because of its direct relation to the specific, real case study considered in this work, which concerns modular GTs: when a GT module is removed, it is sent back to the workshop to undergo a maintenance overhaul, while an AGAN module replaces it on the GT.

Table 1: example of Part Life Data Base $(n=22)$

\begin{tabular}{|l|l|l|l|l|l|}
\hline Record & Time inspec. & \multicolumn{1}{|c|}{$n_{1}$} & $n_{2}$ & $n_{3}$ & $n_{4}$ \\
\hline 1 & 130 & 4 & 5 & 9 & 4 \\
\hline 2 & 180 & 0 & 10 & 11 & 1 \\
\hline & $\cdots$ & $\cdots$ & $\cdots$ & $\cdots$ & $\cdots$ \\
\hline$N$ & 200 & 15 & 5 & 1 & 1 \\
\hline
\end{tabular}

In this context, the aim of this work is to: 
- Apply the MLE technique on the available dataset to infer the values of the parameters of the four-state degradation model.

- Implement a technique to estimate the FIM, which is then used to estimate the confidence intervals on the estimated semi-Markov model parameters.

- Implement a technique for propagating the estimated uncertainties (confidence intervals) onto the probabilities of occupying the degradation states over time. This allows estimating the component future failure behavior and support the business department in the estimation of the costs of maintenance service contracts to be offered to the customers.

\section{Parameter Estimation}

The aim of this section is to determine the analytical expression of the likelihood function $L(\boldsymbol{T} ; \boldsymbol{\theta})$, where the vector $\boldsymbol{T}$ contains the three stochastic transition times $T_{k}, k=1, \ldots, 3$ we are interested in. To do this, we need to compute the probability of finding the component in state $S_{1}$ at inspection time $t_{i}, i=1, \ldots, N$, which is defined as:

$$
P_{1}^{i}=R_{1}\left(t_{i} ; \alpha_{1}, \beta_{1}\right)=P\left(T_{1}>t_{i} ; \alpha_{1}, \beta_{1}\right)
$$

Where $R_{1}\left(t_{i} ; \alpha_{1}, \beta_{1}\right)$ is the Complementary Cumulative Distribution Function (CCDF, also referred to as survival function) of the transition time $T_{1}$, whose parameters are $\alpha_{1}, \beta_{1}$.

The probability $P_{2}^{i}$ of finding the component in state $S_{2}$ at inspection time $t_{i}$ is given by:

$$
P_{2}^{i}=\int_{0}^{t_{i}} f_{1}(\tau) R_{2}\left(t_{i}-\tau ; \alpha_{2}, \beta_{2}\right) d \tau
$$

where $f_{1}(t)$ represents the probability density function (pdf) of the transition time $T_{1}$ from state $S_{1}$ 'Good' to state $S_{2}$ 'Light', whereas $R_{2}\left(t_{i} ; \alpha_{2}, \beta_{2}\right)$ is the CCDF of the transition time $T_{2}$, with parameters $\alpha_{2}, \beta_{2}$.

The probability $P_{3}^{i}$ of finding the component in state $S_{3}$ 'Medium' at inspection at time $t_{i}$ reads:

$$
P_{3}^{i}=\int_{0}^{t_{i}} \int_{\tau_{1}}^{t_{i}} f_{1}\left(\tau_{1}\right) f_{2}\left(\tau_{2}-\tau_{1}\right) R_{3}\left(t_{i}-\tau_{2} ; \alpha_{3}, \beta_{3}\right) d \tau_{2} d \tau_{1}
$$

where $f_{2}(t)$ represents the pdf of $T_{2}$, whereas $R_{3}\left(t_{i} ; \alpha_{3}, \beta_{3}\right)$ is the CCDF of the transition time $T_{3}$, with parameters $\alpha_{3}, \beta_{3}$.

Finally, the probability $P_{4}^{i}$ of finding the component in state Heavy at inspection is derived from the constraint that the four probability values $P_{s}^{i}, s=1, \ldots, 4$ must sum to one:

$$
P_{4}^{i}=1-P_{1}^{i}-P_{2}^{i}-P_{3}^{i}
$$


The parameter estimation problem considered in this work is concerned to inferring the values $\left(\alpha_{k}, \beta_{k}\right)$ of the scale and shape parameters, respectively, of the Weibull distribute transition time $T_{k}$ from state $S_{k}$ to state $S_{k+1}, k=1,2,3$. These parameters are arranged in the vector $\boldsymbol{\theta}=$ $\left(\alpha_{1}, \beta_{1}, \alpha_{2}, \beta_{2}, \alpha_{3}, \beta_{3}\right)$.

The likelihood function is multinomial distribution, which reads:

$$
L(\boldsymbol{T} ; \boldsymbol{\theta})=\prod_{i=1}^{N} \frac{n !}{n_{1}^{i} ! n_{2}^{i} ! n_{3}^{i} !\left(1-n_{1}^{i}-n_{2}^{i}-n_{3}^{i}\right) !}\left(P_{1}^{i}\right)^{n_{1}^{i}}\left(P_{2}^{i}\right)^{n_{2}^{i}}\left(P_{3}^{i}\right)^{n_{3}^{i}}\left(1-P_{1}^{i}-P_{2}^{i}-P_{3}^{i}\right)^{n-n_{1}^{i}-n_{2}^{i}-n_{3}^{i}}
$$

where

- $n_{s}^{i}$ represents the number of components in state $S_{s}$ at inspection time $t_{i}, s=$ $1, \ldots, 4, i=1, \ldots, N$.

- $\quad n$ is the total number of components in the same turbine inspected at each time.

Finally, as usual [18], instead of the likelihood function $L(\boldsymbol{T} ; \boldsymbol{\theta})$, one can maximize the log-likelihood function $l(\boldsymbol{T} ; \boldsymbol{\theta})$, which is its natural logarithm. In fact, $l(\boldsymbol{T} ; \boldsymbol{\theta})$ tends to be simpler than $L(\boldsymbol{T} ; \boldsymbol{\theta})$, as when we take logs, products are changed to sums.

The analytical solution of such MLE problem is impracticable, because the log-likelihood function is made up of convolution integrals, for which there is no solution in closed form. Thus, we first use the adaptive quadrature method ([22]) to numerically calculate the integrals in Eqs (2-3). Then, a combination of optimization algorithms available in the MATLAB Global Optimization toolbox ([25]-[28]) is used to find the maximum of the log-likelihood function. In this respect, notice that the search space in which the optimization algorithm is asked to find the maximum value of the loglikelihood function is very large, as it has $M=6$ dimensions, and there may be local maxima and flat regions. Thus, it may happen that after running any of the optimization algorithms contained in the toolbox (i.e., genetic algorithms, active set method, Nelder-Mead simplex algorithm, etc. [25]-[28]) with a large number of iterations, we get an incorrect ML estimate (a local minimum), or even a Not a Number.

The strategy proposed to overcome this problem is to successively run, for a relatively large number of times (e.g., 5 times), different optimization algorithms. That is, an optimization algorithm is initially run, which provides a first solution in the $6 \mathrm{D}$ solution space. Then, each of these 6 entries is multiplied by a number randomly sampled from $\{0.75,1.25\}$ (i.e., $25 \%$ more or less than the proposed solution). This new, modified point, not far from the starting point, indicates the region of the search space where the MLE values have to be sought, and it is the starting point from which the next, possibly different, optimization algorithm begins the search we take as final optimum point the one 
indicated as optimum by the majority of the algorithm runs. The repeated application of different optimization algorithms starting from different points is done to reduce the risk of finding a local maximum (future research work will focus on the potential use of the ensemble of optimization algorithms for searching the log-likelihood global maximum).

\section{Confidence Intervals}

To estimate the uncertainty in the MLE values, we exploit the asymptotic properties of the ML estimator, which hold under mild regularity conditions [18], [30]. That is, if $\widehat{\boldsymbol{\theta}}=\left(\hat{\theta}_{1}, \ldots, \hat{\theta}_{M}\right)$, with $M \geq 1$ number of parameters to be estimated, is a unique solution to the maximization problem of $l(T ; \boldsymbol{\theta})$, then:

1) $\mathrm{E}(\widehat{\boldsymbol{\theta}}) \rightarrow \boldsymbol{\theta}$

2) $\widehat{\boldsymbol{\sigma}}_{\widehat{\theta}}=\left(\widehat{\boldsymbol{\sigma}}_{\boldsymbol{\theta}_{1}}, \ldots, \widehat{\boldsymbol{\sigma}}_{\boldsymbol{\theta}_{M}}\right) \rightarrow\left(\widehat{\boldsymbol{\sigma}}_{\boldsymbol{\theta}_{1}}, \ldots, \widehat{\boldsymbol{\sigma}}_{\boldsymbol{\theta}_{M}}\right)_{C R}$

where $\left(\widehat{\boldsymbol{\sigma}}_{\boldsymbol{\theta}_{1}}, \ldots, \widehat{\boldsymbol{\sigma}}_{\boldsymbol{\theta}_{\boldsymbol{M}}}\right)_{\boldsymbol{C} \boldsymbol{R}}$ is the Cramer-Rao lower bound of the standard deviations of any unbiased estimator of $\boldsymbol{\theta}$ [18]. That is, any choice of an unbiased estimator of $\boldsymbol{\theta}$ brings a variability in $\widehat{\boldsymbol{\theta}}$ necessarily larger than $\widehat{\boldsymbol{\sigma}}_{\boldsymbol{C R}}$.

3) $\widehat{\boldsymbol{\theta}}$ is asymptotically normal; that is, $\widehat{\boldsymbol{\theta}}-\boldsymbol{\theta} \sim N\left(0, \boldsymbol{F}^{-1}(\widehat{\boldsymbol{\theta}})\right)$.

where $\boldsymbol{F}(\widehat{\boldsymbol{\theta}})$ is the expected FIM calculated at the MLE point. The generic $(m, j)$ entry of $\boldsymbol{F}(\boldsymbol{\theta}), m$, $j=1, \ldots, M$, is:

$$
F_{m j}=E\left[\frac{\partial l(\boldsymbol{T} ; \boldsymbol{\theta})}{\partial \vartheta_{m}} \cdot \frac{\partial l(\boldsymbol{T} ; \boldsymbol{\theta})}{\partial \vartheta_{j}}\right]
$$

Under mild regularity conditions, FIM is equal to the average of the Hessian of $l(\boldsymbol{T} ; \boldsymbol{\theta})$ :

$$
-\left(\begin{array}{ccc}
E\left[\frac{\partial^{2} l(\boldsymbol{T} ; \boldsymbol{\theta})}{\partial \vartheta_{1}{ }^{2}}\right] & \cdots & E\left[\frac{\partial^{2} l(\boldsymbol{T} ; \boldsymbol{\theta})}{\partial \vartheta_{1} \partial \vartheta_{M}}\right] \\
\vdots & \ddots & \vdots \\
E\left[\frac{\partial^{2} l(\boldsymbol{T} ; \boldsymbol{\theta})}{\partial \vartheta_{M} \partial \vartheta_{1}}\right] & \cdots & E\left[\frac{\partial^{2} l(\boldsymbol{T} ; \boldsymbol{\theta})}{\partial \vartheta_{M}{ }^{2}}\right]
\end{array}\right)
$$

Notice that the second derivatives give a measure of how quickly the log-likelihood changes when we change the parameter values in the proximity of the ML estimate. Then, if the Fisher information is large, the distribution changes quickly when we move from the MLE value of the parameter. This entails that we should be able to properly estimate $\widehat{\boldsymbol{\theta}}$ based on the data. On the other hand, if Fisher 
information is small, this means that the distribution is 'very similar' to distributions with parameter not so close to $\widehat{\boldsymbol{\theta}}$ and, thus, more difficult to distinguish; thus, our estimation will be worse.

However, the computation of the FIM in the present case study is even more difficult than that of the MLE, as it requires computing the derivatives of $l(\boldsymbol{T} ; \boldsymbol{\theta})$, for which analytical solutions are not practicable. Thus, the computer resampling approach proposed in [29] is considered in this work to estimate $\boldsymbol{F}(\boldsymbol{\theta})$, which uses a computationally efficient and easy-to-implement method for Hessian estimation.

The underlying idea of the method is to produce a large number of Simultaneous Perturbation (SP) estimates of the Hessian matrix of $l(\boldsymbol{T} ; \boldsymbol{\theta})$ and, then, average the negative of these estimates to obtain an approximation to $\boldsymbol{F}(\boldsymbol{\theta})$ (Figure 2).

The algorithm is presented in detail below:

Step 0. (Initialization). Set the number $\Upsilon$ of pseudo-data vectors that will be generated, and inizialize $v=1$.

Step 1. (Generating pseudo-data). Based on the ML Estimate $\widehat{\boldsymbol{\theta}}$, generate the $\boldsymbol{v}$-th pseudo-dataset $Z_{\text {pseudo }}(v)$ of $N$ records. Namely, to produce the SP Hessian estimates, we implement the following procedure to generate a dataset similar to the original:

1. Set $i=1$.

2. Consider the inspection time of the $i$-th record in the available dataset.

3. Simulate the histories of the $n$ components in the $i$-th record, assuming that the parameters of the stochastic process take their ML estimate $\widehat{\boldsymbol{\theta}}$.

4. Store the outcomes.

5. $i=i+1$.

6. Repeat steps 2-4 until $i>\mathrm{N}$.

Step 2. (Hessian estimation): With the $v$-th pseudo-data vector in Step 1, compute the $\Gamma$ Hessian estimates $\widehat{\boldsymbol{H}}_{\gamma}, \gamma=1 \ldots, \Gamma$. To do this, we first build the perturbation vector $h_{\gamma}=$ $\left(h_{1}^{\gamma}, \ldots, h_{M}^{\gamma}\right)$, whose elements are uniformly distributed zero-mean random variables with bounds satisfying the condition $E\left[\left|\frac{1}{h_{m}^{\gamma}}\right|\right]<\infty, m=1, \ldots, M$. Then, we use the finite difference method [31] for estimating the second derivatives. In particular, the $m$-th element on the diagonal of $\widehat{\boldsymbol{H}}_{\gamma}$ is: 


$$
\begin{gathered}
\frac{\partial^{2} \log L\left(\boldsymbol{\theta} \mid Z_{\text {pseudo }}\right)}{\partial \vartheta_{m}{ }^{2}} \cong \\
\frac{\log \left(\boldsymbol{\theta}_{\vartheta_{m}=\widehat{\vartheta}_{m}+h_{m}^{\gamma}} \mid Z_{\text {pseudo }}\right)-2 \log L\left(\boldsymbol{\theta} \mid Z_{\text {pseudo }}\right)+\log L\left(\boldsymbol{\theta}_{\vartheta_{m}=\widehat{\vartheta}_{m}+h_{m}^{\gamma}} \mid Z_{\text {pseudo }}\right)}{h_{m}^{\gamma^{2}}}
\end{gathered}
$$

The $(m, j)$ elements, $m, j=1, \ldots, M$ and $m \neq j$, outside the diagonal, are:

$$
\begin{aligned}
& \frac{\partial^{2} \log L\left(\boldsymbol{\theta} \mid Z_{\text {pseudo }}\right)}{\partial \vartheta_{m} \partial \vartheta_{j}} \cong \\
& \frac{\log L\left(\boldsymbol{\theta}_{\vartheta_{m}=\widehat{\vartheta_{m}}+h_{m}^{\gamma}, \vartheta_{j}=\widehat{\vartheta_{j}}+h_{j}^{\gamma}} \mid Z_{\text {pseudo }}\right)+\log L\left(\boldsymbol{\theta}_{\vartheta_{m}=\widehat{\vartheta_{m}}-h_{m}^{\gamma}, \vartheta_{j}=\widehat{\vartheta_{j}}-h_{j}^{\gamma}} \mid Z_{\text {pseudo }}\right)}{4 h_{m}^{\gamma} h_{j}^{\gamma}}
\end{aligned}
$$

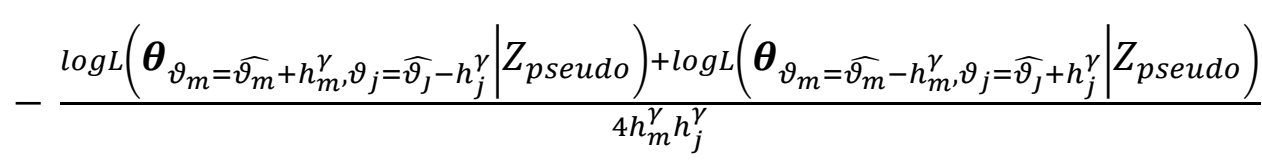

where $\boldsymbol{\theta}_{\vartheta_{m}=\vartheta_{m}^{*}, \vartheta_{j}=\vartheta_{j}^{*}}$ represents vector $\widehat{\boldsymbol{\theta}}$, with its $m$-th and $j$-th elements taking the values $\vartheta_{m}^{*}$ and $\vartheta_{j}^{*}$, respectively.

Finally, the sample mean $\overline{\boldsymbol{H}}^{v}=\overline{\boldsymbol{H}}^{v}\left(\boldsymbol{\theta} \mid Z_{\text {pseudo }}(v)\right)$ of the $\Gamma$ estimates is computed.

Step 3. (Averaging Hessian estimates). Repeat Steps 1 and 2 until $v=\Upsilon$. Then, the negative of the average of the $\Upsilon$ Hessian estimates $\overline{\boldsymbol{H}}^{v}$ produced in Step 2 gives the estimate $\overline{\boldsymbol{F}_{\Gamma \Upsilon}}(\boldsymbol{\theta})$.

The approach is computationally efficient because it requires a low number of log-likelihood values to produce each estimate. 


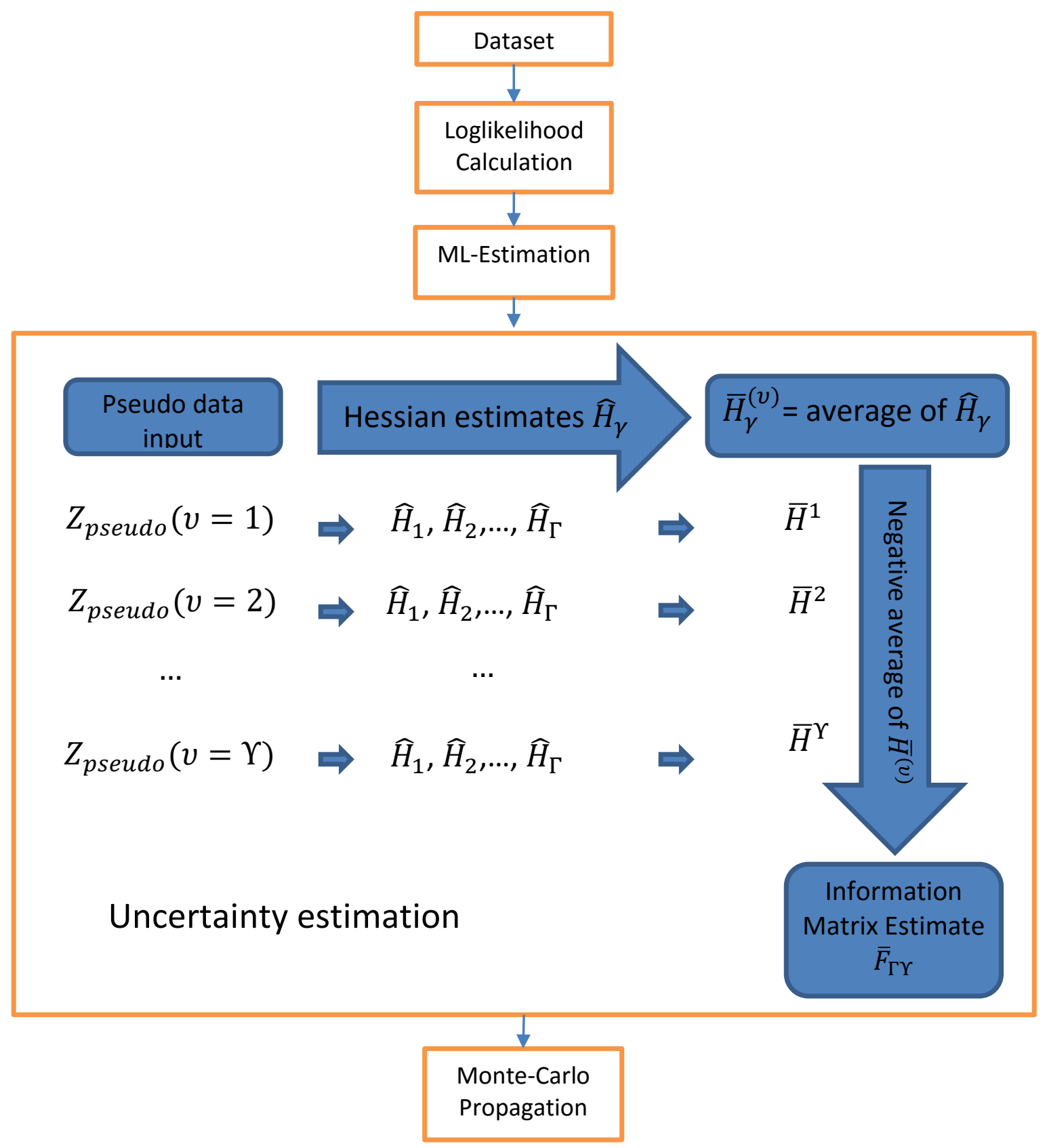

Figure 2: Synoptic of the overall procedure and, in particular, of the bootstrap approach to estimate the Fisher Information Matrix

\subsection{Construction of the confidence intervals}

Approximate confidence intervals for the model parameters can be obtained by using the asymptotic property of the MLE estimators, that is the distribution is normal with means equal to the MLE values and covariance matrix equal to the inverse of the FIM [18]. 
On this basis, an approximate $100(1-\alpha) \%$ normal-theory, Wald confidence region for $\widehat{\boldsymbol{\theta}}$ is the set of all the $\boldsymbol{\theta}$ in the ellipsoid ([32], [33]):

$$
(\widehat{\boldsymbol{\theta}}-\boldsymbol{\theta}) \cdot \boldsymbol{F}^{-1}(\widehat{\boldsymbol{\theta}}) \cdot(\widehat{\boldsymbol{\theta}}-\boldsymbol{\theta})^{\prime} \leq \chi^{2}(1-\alpha, M)
$$

where $\chi^{2}(1-\alpha, M)$ is the $(1-\alpha)^{\text {th }}$ quantile of the chi-square distribution with $M$ degrees of freedom, and $M$ is the number of parameters (i.e., $M=6$ in the considered case study).

On the other hand, for a positive definite parameter (e.g., one of the parameters of the Weibull distribution), the normal approximation is sometimes unsatisfactory because the distribution centred in the MLE values may have the tails in the negative parts of the hyper-space, thus giving negative lower bounds of the confidence intervals. In this case, the delta method [34] is adopted, which improves the quality of the approximation by applying a suitable re-parameterization of the parameters (i.e., a transformation of the parameters to a new scale). The algorithm is presented in detail below.

The delta method states that we can work, instead of $\boldsymbol{\theta}$, with a differentiable non-zero valued function $\phi(\boldsymbol{\theta})$, which is again asymptotically normally distributed around $\phi(\widehat{\boldsymbol{\theta}})$, with adjusted variance value $\widehat{\sigma}^{2}\{\phi(\widehat{\boldsymbol{\theta}})\}=\phi^{\prime}(\widehat{\boldsymbol{\theta}})^{T} \boldsymbol{F}^{-\mathbf{1}}(\widehat{\boldsymbol{\theta}}) \phi^{\prime}(\widehat{\boldsymbol{\theta}})$, where $\phi^{\prime}(\widehat{\boldsymbol{\theta}})$ is the diagonal matrix of the partial derivatives of $\phi$ with respect to the parameters in $\boldsymbol{\theta}$, calculated in $\widehat{\boldsymbol{\theta}}$. That is:

$$
\phi(\boldsymbol{\theta}) \sim N\left(\phi(\widehat{\boldsymbol{\theta}}), \phi^{\prime}(\widehat{\boldsymbol{\theta}}) \boldsymbol{F}^{-\mathbf{1}}(\widehat{\boldsymbol{\theta}}) \phi^{\prime}(\widehat{\boldsymbol{\theta}})^{T}\right)
$$

In this work we consider the function identity for the parameters with positive distribution whereas, in according to [33], $\phi(\cdot)=\ln (\cdot)$ for those parameters with negative tails.

\section{Case Study}

GE offers to its customers a maintenance service on GTs. This service include maintaining the nozzles of the GTs, which are affected by high-temperature oxidation. This mechanism occurs when nickelbased super alloys are exposed to temperatures higher than $538^{\circ} \mathrm{C}$. Oxygen in the gas stream reacts with the nickel alloy to form a nickel-oxide layer on the airfoil surface. When subjected to vibration and start-stop thermal cycles during operation, this nickel oxide layer tends to crack and spall. This phenomenon may also occur on the inner surfaces of blade cooling passages and result in blade failure [12]. 
When nozzles are removed from the turbine system upon a scheduled or opportunistic maintenance action, they are delivered to the GE workshop. There, GE experts check the component and classify its health state into 'Good', 'Light', 'Medium' and 'Heavy' ordinal categories, by comparing the amount of degradation (i.e., the oxidized area) with three thresholds $T_{g}, T_{l}, T_{m}$ :

- If the oxidized area is smaller than $T_{g}$, then the nozzle is not maintained.

- If the oxidized area indicator is larger than $T_{m}$, then the component is replaced.

- If the oxidized area is between $T_{g}$ and $T_{l}$, then a maintenance action based on brazing is performed.

- If the oxidized area is between $T_{l}$ and $T_{m}$, then a welding repair action is performed.

Depending on the degradation state, different workshop activities are carried out to send back to the customer the nozzle system in an As Good As New (AGAN), or even new, state.

A number of physical models have been proposed to describe the behaviour of the oxidation mechanism, which emphasize the dependence of the degradation evolution on the type of substance that creates oxidation ([37], [38]). This information is at the basis of the development of the proposed multi-state degradation model, to describe the dynamics of stochastic transition among the 4 degradation states identified by the maintenance operators. Given the cumulative, incremental nature of the degradation process under study, we assume that the transitions in the model can occur from one degradation state to the next degraded state, only, and, as mentioned before, at Weibulldistributed stochastic time instants.

The information about the nozzle system operating life and the result of the workshop classification are stored in a Part Life Data Base (PLDB) for reliability life data analysis. In particular, with regards to the oxidation mechanism, the PLDB contains a set of $N=30$ records of field observations of the nozzle systems of turbines operated by GE. We anticipate that the data considered in this paper came from real GE field experience, but they have been re-scaled in arbitrary time units to respect the GE policy on confidential information.

Table 2 reports the ML estimate of $\boldsymbol{\theta}$ (second column) and the parameters on which the delta method has been applied (third column). That is, in this case study $\phi(\boldsymbol{\theta})=\left[\ln \left(\alpha_{1}\right), \beta_{1}, \ln \left(\alpha_{2}\right), \beta_{2}, \alpha_{3}, \beta_{3}\right]$, and the diagonal of $\phi^{\prime}$ is $\operatorname{diag}\left(\phi^{\prime}(\boldsymbol{\theta})\right)=\left[1 / \alpha_{1}, 1,1 / \alpha_{2}, 1,1,1\right]$. The fourth column gives the value of the standard deviations, which are the square roots of the elements of the diagonal of $\boldsymbol{F}^{\mathbf{- 1}}(\widehat{\boldsymbol{\theta}})$. Finally, the last two columns show the $90 \%$ confidence bounds of $\widehat{\boldsymbol{\theta}}$. To find these values, we have used the common formula $\phi(\widehat{\boldsymbol{\theta}}) \pm Z_{\frac{\alpha}{2}} \cdot \widehat{\sigma}\{\phi(\widehat{\boldsymbol{\theta}})\}$, for estimating the equal-tails $1-\alpha$ confidence 
interval for $\phi(\widehat{\boldsymbol{\theta}})$, i.e., on the re-parametrized scale. Then, we can get the confidence bounds of $\widehat{\boldsymbol{\theta}}$ by inverting $\phi(\cdot)$, i.e., converting to the original scale. For example, for the first parameter we have $e^{\ln \left(\widehat{\alpha_{1}}\right) \pm z \frac{\alpha}{2} \cdot \frac{\widehat{\sigma}\left\{\hat{\alpha}_{1}\right\}}{\widehat{\alpha}_{1}}}$

\begin{tabular}{|cccccc|}
\hline Parameters & $\begin{array}{c}\text { ML } \\
\text { estimation }\end{array}$ & reparametrization & Std dev & Lower & Upper \\
bound & bound \\
\hline $\boldsymbol{\alpha}_{\mathbf{1}}$ & 12620 & Yes & 8509.8 & 4162.5 & 38261 \\
\hline $\boldsymbol{\beta}_{\mathbf{1}}$ & 0.1062 & No & 0.0135 & 0.0840 & 0.1284 \\
\hline $\boldsymbol{\alpha}_{\mathbf{2}}$ & 109.8512 & Yes & 73.9670 & 36.2916 & 332.5091 \\
\hline $\boldsymbol{\beta}_{\mathbf{2}}$ & 0.0950 & No & 0.0159 & 0.0688 & 0.1212 \\
\hline $\boldsymbol{\alpha}_{\mathbf{3}}$ & $3.3650 \mathrm{e} 3$ & No & 1096.7 & 1561.1 & 5168.9 \\
\hline $\boldsymbol{\beta}_{\mathbf{3}}$ & 0.3480 & No & 0.0699 & 0.2331 & 0.4630 \\
\hline
\end{tabular}

Table 21: Parameters estimation: results

The uncertainty in the model parameters is, then, propagated via a double-loop Monte Carlo simulation approach [35] to estimate the CDFs of the probabilities of finding the nozzle in the degradation states (i.e., a plot for each degradation state) for every time instant of the discretized time horizon.

In details, the procedure entails sampling a six dimension vector of numbers from $\phi(\boldsymbol{\theta}) \sim N\left(\phi(\widehat{\boldsymbol{\theta}}), \phi^{\prime}(\widehat{\boldsymbol{\theta}})^{T} \boldsymbol{F}^{-\mathbf{1}}(\widehat{\boldsymbol{\theta}}) \phi^{\prime}(\widehat{\boldsymbol{\theta}})\right)$; this is the outer loop. Then, for each of these samples, the Monte Carlo simulation, the inner loop, is used to estimate the probability over time of finding the nozzle in state $S_{s}$, with $s=1, \ldots, 4$. Finally, the gathered evidence is used to compute at every time instant the CDFs of the probabilities of finding the nozzle in the four states.

For example, Figure 3 shows the results relevant to state $S_{1}$ at time instant $t=5000$ Units of Time (UoT). The practical interpretation of the Figure can be: the probability of the nozzle being in state $S_{1}$ at $t=5000$ cannot be smaller than 0.3 ; it is smaller than 0.4 with probability 0.5 ; certainly, it is smaller than 0.5. Another example is reported in Figure 4. The probability that the nozzle is in the

\footnotetext{
${ }^{1}$ For every model parameter (first column), the MLE value is reported (second column). On the third column, it is indicated whether the delta method has been applied. The estimated standard deviation is reported on the fourth column, whereas the lower and upper bounds of the confidence interval on the MLE value are reported in the fifth and sixth columns, respectively
} 
fourth state at time $t=5000$ UoT is smaller or equal to 0.46 with probability almost 1 . Similar plots are produced for $S_{2}$ and $S_{3}$.

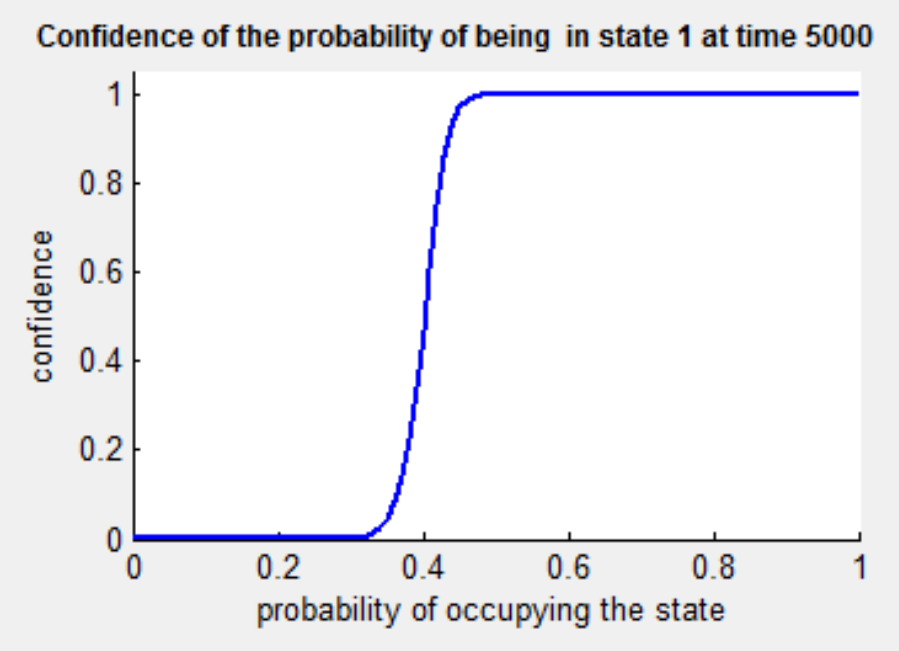

Figure 3: CDF of the probability of state $S_{1}$ at $\mathrm{t}=5000 \mathrm{UoT}$

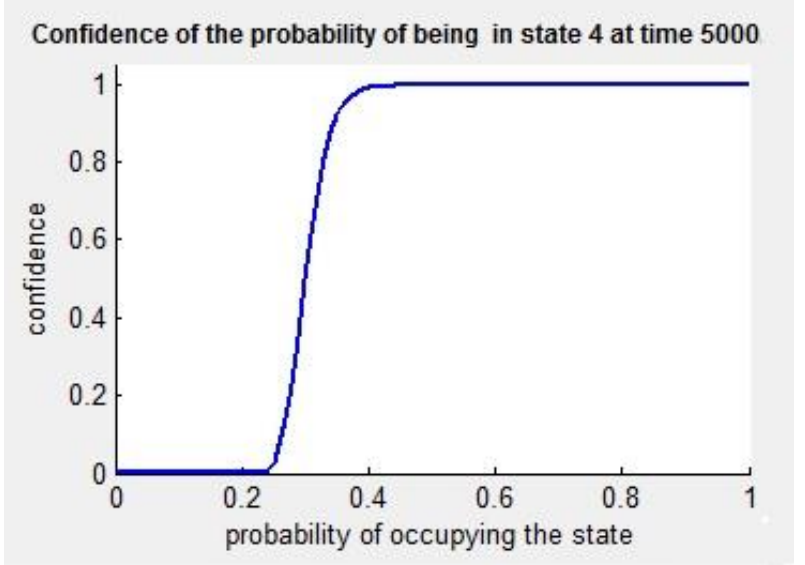

Figure 4: CDF of the probability of state $S 4$ at $t=5000$ UoT

Figure 4 shows the link between the CDF of the state probability at a given time instant and the timedependent state probability, with associated confidence interval. 

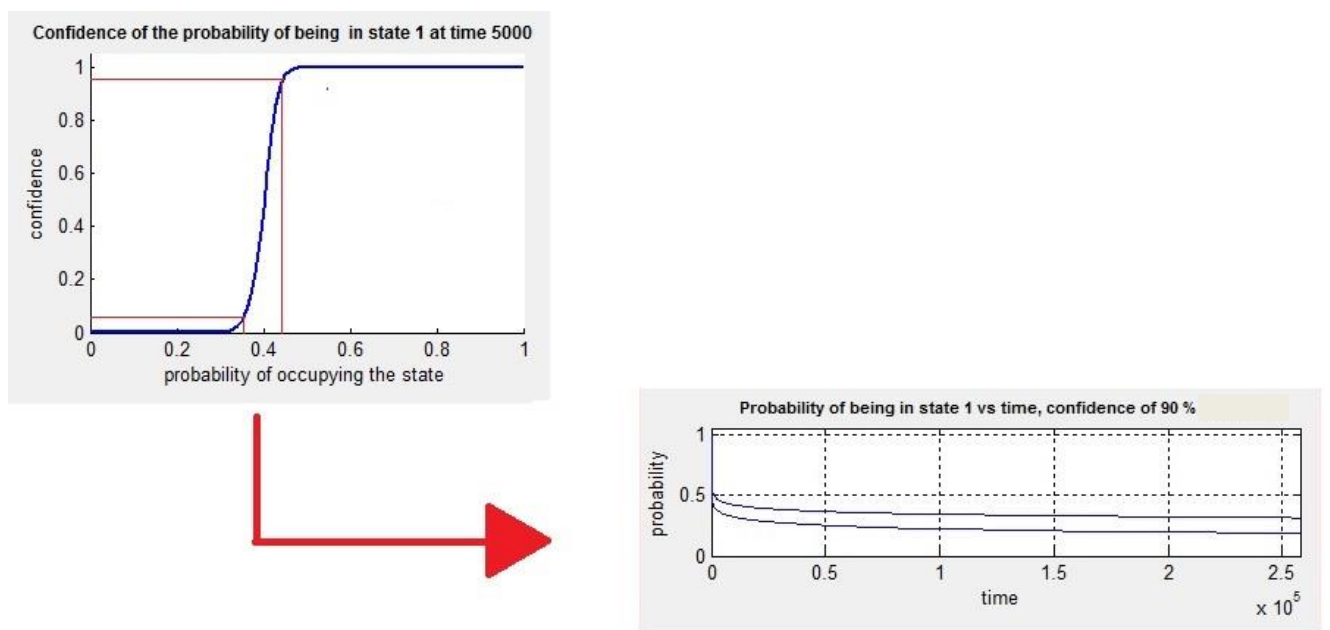

Figure 5: Link between CDF of the state probability at a specific time instant and time-depended state probability, with associated confidence interval of $90 \%$. The extreme points of this interval (i.e., $5^{\text {th }}$ and $95^{\text {th }}$ percentiles) are reported on the time axis.

Figure 6 shows the time-depended probability values of the nozzle being in the four degradation states, which is useful information to make decisions. It emerges that:

- In the initial part of the component life, the items are healthy, with probability of finding the nozzle in state 1 at time 0 equal to 1 .

- In the successive time instants, the nozzle starts to increase the probability of leaving state $S_{1}$ to reach state $S_{2}$; the first three plots in Figure 6 show that the sojourn times in states $S_{1}, S_{2}$ and $S_{3}$ are on average very small, with a large variance, due to the small values of the shape parameters.

- As time goes on, the probability that the nozzle undergoes a transition from state $S_{2}$ to state $S_{3}$ does not significantly increase.

- Finally, as the time evolves, the nozzle reaches the failure state.

From these considerations, it emerges that further practical work must be spent on investigating whether the degradation mechanism should be modelled as a three-state system. In fact, the smaller number of parameters to be estimated may provide parameter MLE affected by a small amount of uncertainty. However, this investigation does not concern looking at the results that one would get if two of the four states were merged together; rather, GE engineers should find two new threshold values for the oxidized area, based on their physical knowledge of the degradation phenomenon: once a PLDB with respect to these new threshold values is available, then a 3-state system model can be developed. 

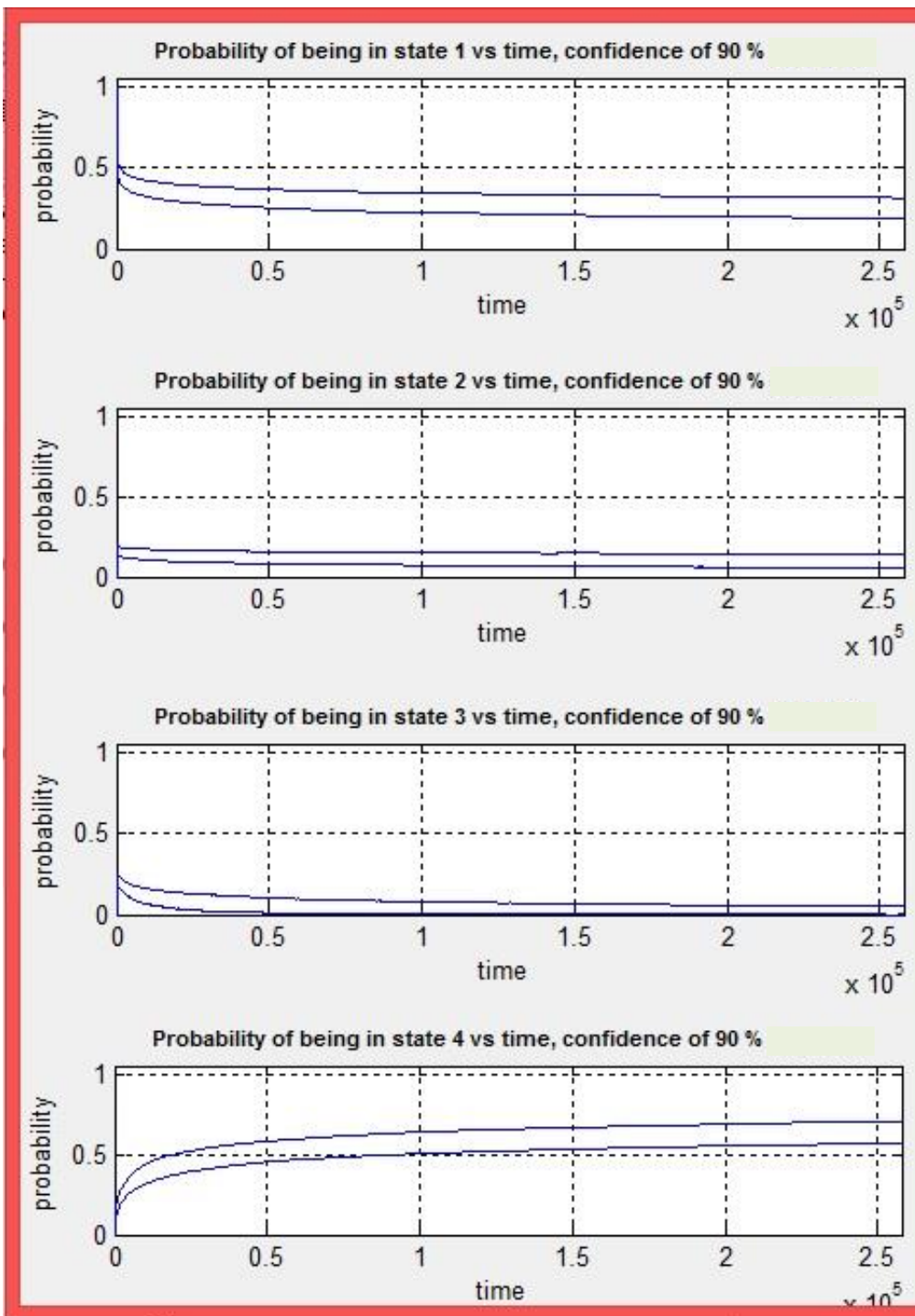

Figure 6: Time-depended state probability, with associated confidence interval of $90 \%$.

The output of the proposed procedure, summarized in Figure 6, can be exploited in approaches like those developed by the authors to optimize CBM strategies ([20], [21]) based on multi-state models of degradation, with uncertain parameters. In particular, two techniques based on Multi Objective Genetic Algorithms have been proposed in [21] to solve the problem of the optimal inspection scheduling within a CBM strategy. Both techniques rely on an innovative algorithm for sorting probability distributions and on a new definition of Pareto dominance. The first technique is based on the NSGA-II algorithm, whereas the second is a combination of NSGA-II and the CVaR index. Yet, a similar solution has been proposed in [20], where the uncertainty is handled within the framework of the Dempster-Shafer theory of evidence. From these studies, it also emerges the added value of giving due account to uncertainty when making maintenance decisions: it gives the 
possibility of choosing maintenance settings which would be otherwise discarded if the decision maker looks at the expected values only.

\section{Conclusion}

In this paper, a methodology to build a four-state semi-Markov model of degradation has been developed. In particular, the methodology proposed allows estimating the model parameters, characterizing the related uncertainty and propagating it onto the probabilities of occupying the different states over time.

This output has paved the way for the development of advanced computational techniques to optimize maintenance policies in the presence of uncertainty and imprecisions.

A practical case study has been considered, which concerns the oxidation degradation mechanism that affects the nozzles of gas turbines. The case study has shown the potential of the method and, at the same time, has uncovered the practical possibility of describing the degradation process in three states.

Future research work will focus on the development of Bayesian methods for estimating the parameters of the multi-state degradation model.

\section{Reference}

[1].Baraldi, P., Balestrero, A., Compare, M., Benetrix, L., Despujols, A., Zio, E., “A modeling framework for maintenance optimization of electrical components based on fuzzy logic and effective age", Quality and Reliability Engineering International. Vol. 29, No. 3, pp. 385-405, 2013.

[2]. Baraldi, P., Compare, M., Zio, E., "Uncertainty analysis in degradation modeling for maintenance policy assessment", Proceedings of the Institution of Mechanical Engineers, Part O: Journal of Risk and Reliability, Vol. 227, No. 3, pp. 267-278, 2013.

[3]. Baraldi P., Compare, M., Zio, E., "Maintenance policy performance assessment in presence of imprecision based on Dempster-Shafer Theory of Evidence”, Information Sciences. Vol. 245, pp. 112-131, 2013.

[4].Baraldi, P., Compare, M., Zio, E., "A practical analysis of the degradation of a nuclear component with field data, in Reliability and Risk analysis: Beyond the Horizon", Eds. R.D.J.M. Steenbergen, P.H.A.J.M. van Gelder, S. Miraglia, A.C.W.M. Vrouwenvelder, CRC Press, 2013.

[5]. Baraldi, P., Compare, M., Despujols, A., Zio, E., "Modelling the effects of maintenance on the degradation of a water-feeding turbo-pump of a nuclear power plant", Proceedings of the 
Institution of Mechanical Engineers, Part O: Journal of Risk and Reliability, Vol. 225, No. 2, pp. 169-183, 2011.

[6].Moghaddass, R., Zuo, M.J., “A parameter estimation method for a multi-state deteriorating system with incomplete information", Proceeding of the Seventh International Conference on Mathematical Methods in Reliability: Theory, Methods, and Applications (MMR 2011), pp. 34-42, 2011.

[7].Moghaddass, R., Zuo, M.J., "Multi-state degradation analysis for a condition monitored device with unobservable states", Proceedings of 2012 International Conference on Quality, Reliability, Risk, Maintenance, and Safety Engineering, ICQR2MSE 2012, pp. 549-554, 2012.

[8].Levitin, G., Lisnianski, A., Ushakov I. "Reliability of Multi-State Systems: A Historical Overview," Mathematical and statistical methods in reliability, Lindqvist, Doksum (eds.), World Scientific, pp. 123-137, 2003.

[9].Zuo, M. J., Huang, J. and Kuo, W. "Multi-state k-out-of-n Systems," Reliability Engineering Handbook, (H. Pham, ed.), Springer-Verlag, London, September 2001.

[10]. Vrignat, P., Avila, M., Duculty, F., Aupetit, S., Slimane, M., Kratz, F. "Maintenance policy: degradation laws versus Hidden Markov Model availability indicator," Proceedings of the Institution of Mechanical Engineers, Part O: Journal of Risk and Reliability Vol. 226, No. 2, pp. 137-155, 2011.

[11]. Lisnianski, A., Levitin, G. "Multi-State System Reliability: Assessment, Optimization and Applications," Series on Quality, Reliability and Engineering Statistics: Volume 6, World Scientific, 2003.

[12]. Greenfield, P., "Creep of Metals at High Temperatures," M\&B Monograph ME/9, Mills and Boon Ltd., London, England, 1972.

[13]. Fleming, K.N., "Markov models for evaluating risk-informed in-service inspection strategies for nuclear power plant piping systems," Reliability Engineering and System Safety, Vol. 83, No. 1, pp. 27-45 2004.

[14]. Di Maio, F., Colli, D., Zio, E., Tong, J., "A multi-state Approach for the Reliability Assessment of Nuclear Power Plants Piping Systems, submitted for publication.

[15]. Zio, E., Compare, M., "Evaluating maintenance policies by quantitative modeling and analysis”, Reliability Engineering and System Safety, Vol. 109, pp. 53-65, 2013.

[16]. Zio, E., Compare, M., “A snapshot on maintenance modeling and applications. Marine Technology and Engineering”, Vol. 2, pp. 1413-1425, 2013. 
[17]. Zio, E., "Computational Methods for Reliability and Risk Analysis”, World Scientific Publishing, Singapore, 2009.

[18]. Papoulis, A. And Pillai S.U. 2002. "Probability, Random Variables and Stochastic Processes", $4^{\text {th }}$ edition, International Edition, Mc-Graw-Hill.

[19]. Efron B., Tibshirani R.J. "An introduction to the bootstrap”, Chapman \& Hall, New York, 1993.

[20]. Compare, M., Zio, E., "Genetic algorithms in the framework of Dempster-Shafer theory of evidence for maintenance optimization problems," IEEE Transactions on Reliability, Vol. 64, No. 2, pp. 645-660, 2015.

[21]. Compare, M., Martini, F., Zio, E., "Genetic algorithms for condition-based maintenance optimization under uncertainty,” European Journal of Operational Research, Vol. 244, No. 2, pp. 611-623, 2015.

[22]. Gander, W. and W. Gautschi, “Adaptive Quadrature - Revisited,” BIT, Vol. 40, pp. 84-101, 2000.

[23]. Christensen, R., Johnson, W., Branscum, A., Hanson, T., "Bayesian Ideas and Data Analysis: An Introduction for Scientists and Statisticians," CRC Press, 2010.

[24]. Di Maio, F., Compare, M., Mattafirri, S., Zio, E. “A double-loop Monte Carlo approach for Part Life Data Base reconstruction and scheduled maintenance improvement," Safety and Reliability: Methodology and Applications - Proceedings of the European Safety and Reliability Conference, ESREL 2014, pp. 1877-1884, 2015.

[25]. Lagarias, J.C., Reeds, J. A., Wright, M. H., Wright, P.E., "Convergence Properties of the Nelder-Mead Simplex Method in Low Dimensions," SIAM Journal of Optimization, Vol. 9, No. 1, pp. 112-147, 1998.

[26]. Powell, M.J.D., “A Fast Algorithm for Nonlinearly Constrained Optimization Calculations," Numerical Analysis, ed. G.A. Watson, Lecture Notes in Mathematics, Springer-Verlag, Vol. 630, 1978.

[27]. Powell, M.J.D., "The Convergence of Variable Metric Methods For Nonlinearly Constrained Optimization Calculations," Nonlinear Programming 3 (Mangasarian, Meyer, and Robinson, eds.), Academic Press, 1978.

[28]. Waltz, R.A., Morales, J.L., Nocedal, J., Orban, D., “An interior algorithm for nonlinear optimization that combines line search and trust region steps," Mathematical Programming, Vol. 107, No. 3, pp. 391-408, 2006. 
[29]. Spall, J.C. "Monte Carlo Computation of the Fisher Information Matrix in Nonstandard Settings", Journal of Computational and Graphical Statistics, Vol. 14, No. 4, pp. 889-909, 2005.

[30]. Kendall, M. and Stuart A., "The Advanced Theory of Statistics," Vol. 2, Charles Griffin and Company limited, London \& High Wycombe, 1979

[31]. Quarteroni, A. "Modellistica Numerica per Problemi Differenziali", 4th Edition, Springer 2008.

[32]. Cox, D.R., Hinkley, D. V., "Theoretical Statistics”, Chapman and Hall, London, 1974. [33]. Meeker, W.Q., Escobar, L.A., "Teaching About Approximate Confidence Regions Based on Maximum Likelihood Estimation”, The American Statistician, Vol. 49, No. 1, pp. 48-53, 1995.

[34]. Giorgio, M., Guida, M., Pulcini, G., “An age- and state-dependent Markov model for degradation processes”, IIE Transactions, Vol. 43, No. 9, pp. 621-632, 2011.

[35]. Zio, E., "The Monte Carlo Simulation Method for System Reliability and Risk Analysis", Springer, 2013.

[36]. Zio, E., "An introduction to the basics of reliability and risk analysis", WorldScientific Publishing Company, Singapore, 2007.

[37]. Wood, I.H., Foster, A.D., Schilke, P.W., "High Temperature Coating for Improved Oxidation/Corrosion Protection," GE Turbine Reference Library Publication GER 3597, 1990.

[38]. Kurz, R., Brun, K., "Degradation in Gas Turbine System”, The American Society Of Mechanical Engineers, ASME paper 2000-GT-345. 